\title{
Assessing the Likelihood of Rare Medical Events in Astronauts
}

\author{
Jerry G. Myers, Jr., Ph.D NASA Glenn Research Center \\ Beth E. Lewandowski, Ph.D NASA Glenn Research Center \\ John E. Brooker, NASA Glenn Research Center \\ Aaron S. Weaver, Ph.D NASA Glenn Research Center
}

Key Words: Probabilistic modeling, space medical events, astronaut health, medical risk analysis

\section{SUMMARY \& CONCLUSIONS}

Despite over half a century of manned space flight, the space flight community is only now coming to fully assess the short and long term medical dangers of exposure to reduced gravity environments. Further, as new manned spacecraft are designed and with the advent of commercial flight capabilities to the general public, a full understanding of medical risk becomes even more critical for maintaining and understanding mission safety and crew health. To address these critical issues, the National Aeronautics and Space Administration (NASA) Human Research Program (HRP) has begun to address the medical hazards with a formalized risk management approach by effectively identifying and attempting to mitigate acute and chronic medical risks to manned space flight.

This paper describes NASA Glenn Research Center's (GRC) efforts to develop a systematic methodology to assess the likelihood of in-flight medical conditions. Using a probabilistic approach, medical risks are assessed using well established and accepted biomedical and human performance models in combination with fundamentally observed data that defines the astronauts' physical conditions, environment and activity levels. Two different examples of space flight risk are used to show the versatility of our approach and how it successfully integrates disparate information to provide HRP decision makers with a valuable source of information which is otherwise lacking.

\section{INTRODUCTION}

The identification of in-flight medical risk in astronauts is usually assessed in two ways. The first is when the medical event has been observed to occur in a previous space flight. In-flight nausea and headaches are examples of frequently occurring conditions requiring medical attention. Likelihood of occurrence and clinical outcome of these events during future missions can be derived from the rate and impact of the medical event's occurrence during prior missions. The second way is from informed, expert opinion identification of potential medical events, even if such events have not been observed in US astronauts during a space mission. Examples of these would be a skeletal fracture and the presentation of a renal stone leading to renal colic. The likelihood in these later cases can sometimes be estimated from terrestrial data, provided appropriate analog populations are available. Unfortunately this is not always feasible, especially when gravity or other flight conditions play an important role in the clinical scenario.

One of the more advanced tools employed by HRP to assess the relative impact of medical events to the crew and mission is the Integrated Medical Model (IMM) [1]. IMM is a probabilistic, simulation-based tool designed to help determine the relative risk associated with medical conditions that could occur in space and to optimize mitigations tools, such as the in flight medical kit. IMM utilizes probabilistic risk assessment (PRA) methods in combination with the best available evidence and current understanding of medical outcomes to predict the rate of occurrence and the resources needed for a successful response to an in-flight medical event.

IMM's primary forecast methodology is insufficient for events that have not yet occurred in space, but are still considered a potential hazard to space flight. In this case, the IMM model relies on an external analysis based on more fundamental observations and physical principles. A team at NASA GRC supports IMM by developing these models that forecast medical event likelihood from first principle and root cause information. To accomplish this task the IMM team at NASA GRC has developed an approach that utilizes well established PRA methods from engineering in combination with the evolving techniques in biomechanics, physiology and human performance modeling to assess the likelihood of specific medical events. The methodology is described in detail and implementation is illustrated in two important clinical scenarios: wrist fracture and sleep disruption requiring medical intervention.

\section{APPROACH}

The approach employed at NASA GRC increases the fidelity of IMM by integrating probabilistic analysis with deterministic models. This approach, new to the medical field, takes its application direction from many engineering fields, including fault tree analysis, simulation-based PRA and deterministic model uncertainty analysis. The unique aspect is how these techniques are integrated with the astronauts' 
overall condition to estimate the likelihood of injury, physiological system failure or environmental impact on the astronaut.

The approach to developing a model of medical event likelihood in the space environment, in the absence of directly observed event occurrences, proceeds along the following general steps:

1) A clinical event of concern is identified

2) The space mission parameters that place the clinical event in context are defined from medical operations personnel

3) A thorough literature review is performed to identify:

a) The medical, environmental and performance parameters critical to defining the process of the medical event.

b) The primary metrics that define the occurrence of the medical event.

c) Biomechanics, physiology or performance deterministic models that most effectively integrate the contributing parameters in the calculation of the primary metrics.

4) Data from the literature and from in-flight observed conditions are used to define population distributions for each of the medical, environmental and performance parameters.

5) Information from 2- 4 is used to develop a PRA (Monte Carlo) model that utilizes the deterministic model identified in 3c as a fundamental integrating mechanism to provide the link between observed medical parameters and the primary metrics.

From an engineering perspective, the above would serve to provide substantial insight into the likelihood of the medical risk provided well defined thresholds of the primary metrics that define when the medical event occurs can be established. Unfortunately, the current understanding of human physiology and performance is not as well defined as failure modes of engineering materials and mechanisms, leading to an extensive uncertainty in the occurrence thresholds. It is necessary to take additional steps to assess the probability of medical event occurrence from our estimates of the integrated metrics describing the medical events. This is most often performed using logistic regression to generate functional transformations of the primary metric to a probability of occurrence. Once addressed in this manner, the output of the model is a probability density function (PDF) describing the estimated number of events per-mission or the most likely probability of occurrence within the mission time frame, with associated uncertainty as defined by the data and model structure.

\section{METHODOLOGY AND APPLICATION}

When addressing a particular medical event sequence, augmentation to the general approach structure is required to accommodate the unique aspects of the specific problem. The team at NASA GRC has been particularly successful in addressing injury modalities such as bone fracture [2] on planetary missions. Here we present two examples of how our general approach was applied to specific medical event analyses. The first is an analysis of the probability of wrist fracture on the International Space Station (ISS). The second is prediction of the rate of medication use to combat the effects of circadian sleep disruption during space flight.

\subsection{Wrist fracture}

There is a legitimate concern with regard to skeletal bone fracture during space missions. A bone fracture can be considered a structural failure of the bone that occurs when the load placed upon the bone exceeds its structural strength [3]. Apparent bone strength is dependent on several factors including mineral content, prior microdamage, geometry, architecture, age and the nature of the applied load [4]. Loading that exceeds the strength of the bone can occur during an accident where a high impact load is experienced. The fracture risk index (FRI) is the ratio of the apparent bone strength to the skeletal loading. A low FRI indicates a low probability of fracture, while a high FRI $(>1)$ indicates a fracture is almost certain [5].

Unexpected or off nominal events, exacerbated by the state of an astronauts weakened bones due to spaceflight induced loss of bone mineral density [6], resulting in fracture, are a real and present danger to space flight success. Additionally, space missions are severely constrained in resources, and by their very nature, provide limited access to medical care. This can have a serious impact and lead to loss of mission or crew member. Since the possibility of fracture exists and the impact to the mission could be substantial, it is crucial to quantify the risk of bone fracture during space exploration missions so that mitigation strategies can be engineered.

Previous efforts at NASA GRC to quantify bone fracture risk on moon and Mars missions have illustrated that, while substantial bone mineral can be lost at load bearing locations, it is the wrist which has the highest probability of fracture due to forceful impacts such as falls [2]. The likelihood of wrist fracture during an ISS mission was also assessed. Our general modeling approach for medical events was applied to address wrist loading during a push off, impact or other sudden deceleration involving the wrist and shoulder after translation across the ISS cabin. The following steps outline the model:

1) Determine the appropriate representation of the dynamic response to external loading for our application. Estimate the skeletal strength at the wrist for this situation from literature data.

2) Determine the skeletal loading from a mass-springdamper, biomechanical model of the wrist, arm and shoulder. Modulate loading with specifics conditions related to the ISS environment.

3) Estimate the probability of loading event occurrence during each mission day, based on the incidence rate of ISS translations, from observed data from ISS mission history.

4) Develop a transfer function that translates FRI to a 
probability of fracture, using observational, clinical data of terrestrial wrist fracture.

5) With a Monte Carlo simulation, develop a PDF of the probability of a loading event and a PDF of the probability of fracture from the loading event. Calculate the overall probability of wrist fracture per mission day by combining the two PDF's.

Step 1 was satisfied with accrued data describing skeletal wrist strength for the impact dynamics assumed aboard the ISS and from appropriate astronaut analog populations [7;8].

The biomechanical model of step 2 is illustrated in Figure 1. Differential equations of motion, described in detail in [2], were solved to determine the load on the wrist during an impact with an ISS wall. The velocity of impact was determined from four studies of the force resulting from a push-off of a space craft wall in a microgravity environment [9-13].The peak forces reported in these studies, along with the subjects' masses were used with a derived force pulse width $(1.1 \pm 20 \%$, based on the average push-off force duration in [9]) to estimate impact velocity. A distribution was developed from these data and from the solution of the biomechanical model for the peak force during an impact for subjects with an average mass of approximately $73 \mathrm{~kg}$. The force was then scaled to a body mass that was based on anthropometric data from the astronaut corps. The calculated peak force was also modulated to account for the fact that impact force decreases with increasing elbow flexion. A uniform distribution of elbow flexion $\left(150-180^{\circ}\right)$ was used in our model and the average decrease in impact force per degree of flexion used was $1.4 \pm 1.4 \% /{ }^{\circ}$ [14-16].

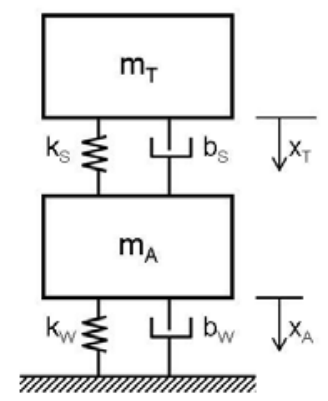

Figure 1. Biomechanical mass-spring-damper model for wrist impact. The total body mass minus the arm mass $\left(\mathrm{m}_{\mathrm{T}}\right)$ and arm mass $\left(\mathrm{m}_{\mathrm{A}}\right)$; the stiffness of the shoulder $\left(\mathrm{k}_{\mathrm{S}}\right)$ and wrist $\left(\mathrm{k}_{\mathrm{W}}\right)$; the damping characteristics of the shoulder $\left(\mathrm{b}_{\mathrm{S}}\right)$ and wrist $\left(\mathrm{b}_{\mathrm{W}}\right)$; and the displacement of $\mathrm{m}_{\mathrm{T}}\left(\mathrm{x}_{\mathrm{T}}\right)$ and $\mathrm{m}_{\mathrm{A}}\left(\mathrm{x}_{\mathrm{A}}\right)$ are shown.

For step 3, a database of in-flight musculoskeletal injuries provided a means to estimate the loading incidence rate. Five of 219 musculoskeletal injuries during the 231,724.7 hours of in-flight operations by U.S. Astronauts have occurred at the wrist [17]. This equates to $5.18 \times 10^{-4}$ wrist injuries per day, which was used in our analyses, along with an estimated $10 \%$ standard deviation derived from expert opinion. Since the incidence rate only takes into account events where an injury could occur, the impact force was represented as a force which was capable of producing an injury. No data exists on injury capable force vs. nominal force. Instead, an assumption was made that the mean injury capable force is equal to the 90th percentile of the normal force.

A logistic regression was performed to develop the transfer function of step 4. Terrestrial data was used to link estimates of FRI to fracture probability by comparing the binary condition of actual fractures to non-fractures, with reference to a set of appropriate controls and specified loading events [18]. The result of the regression was a sigmoidal curve that related FRI to probability of fracture [2].

As described in step 5, A Monte Carlo simulation was performed to assess the likelihood of wrist fracture during a 6 month ISS mission. The result of running the simulation 100,000 times was the FRI distribution shown in Figure 2. It is noted that the 95th-percentile FRI does not exceed 0.5, indicating that the load is less than the bone strength most of the time. The PDF for probability of fracture is illustrated in Figure 3. The mean likelihood for wrist fracture is very low, although the uncertainty, as represented by the width of the standard deviation is significant. The results are also tabulated in Table 1.

TABLE 1. SUMMARY OF RESULTS

\begin{tabular}{|c|c|c|c|c|}
\hline & Mean & $\begin{array}{c}\text { Standard } \\
\text { Deviation }\end{array}$ & $5 \%$ & $95 \%$ \\
\hline FRI & 0.23 & 0.68 & 0.05 & 0.49 \\
\hline $\begin{array}{c}\text { Fracture } \\
\text { Probability }\end{array}$ & $1.2 \times 10^{-5}$ & $6.4 \times 10^{-5}$ & $1.8 \times 10^{-13}$ & $3.2 \times 10^{-5}$ \\
\hline
\end{tabular}

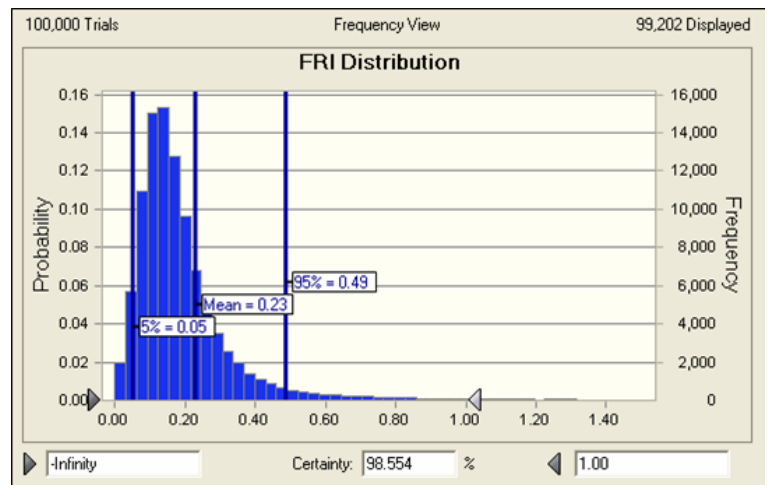

Figure 2. The FRI distribution used to calculate the probability of wrist fracture during an ISS mission. 


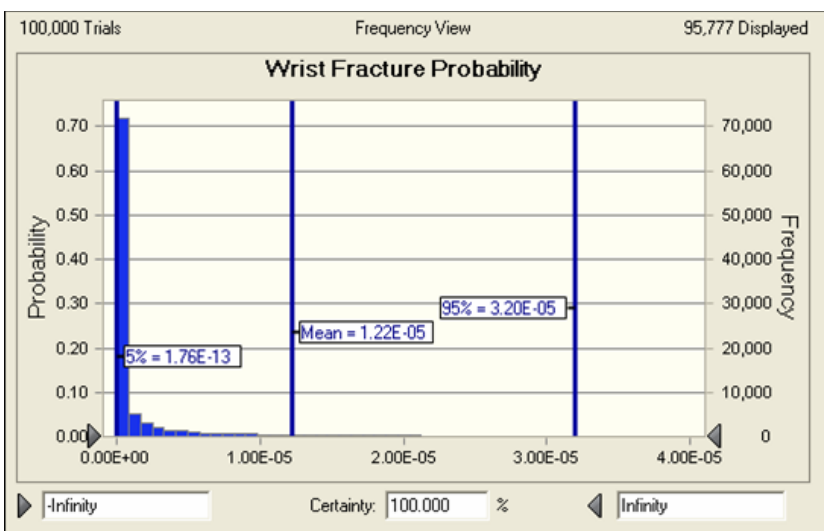

Figure 3. The probability distribution of the probability of wrist fracture during an ISS mission.

\subsection{Estimating the Level of Sleep Medication Use}

During ISS missions, difficulties with sleep affect more than half of all US crews. Mitigation strategies to help astronauts cope with the challenges of disrupted sleep patterns can have a substantial impact on vehicle specific consumable mass or volume or on the mission timeline.

Developed in partnership with collaborators at the Air Force Research Laboratory and Institutes for Behavior Resources (IRB), Inc., the Sleep Disruption and Medical Intervention Forecasting (SDMIF) tool was designed to estimate the occurrence and impact of sleep disturbance with regard to mission planning and the need for medical interventions. The module concept (Figure 4) includes a representative mission sleep and wake schedule that was based on scenario design, mission parameters and historical patterns of events that effect astronaut sleep schedules. This information was used to estimate metrics representing the need for sleep, which were then compared to threshold values to diagnose the likelihood for treatment. The metrics were correlated to the likelihood that a consumable mitigation approach was used during a particular mission day. The specific steps used to accomplish this process are as follows:

1) Generate a daily schedule of crew sleep opportunities for a simulated ISS increment

2) Convert sleep opportunities to actual sleep times

3) Generate a sleep quality factor

4) Pass the sleep/wake period parameters to the Sleep, Activity, Fatigue, and Task Effectiveness (SAFTE) model to evaluate astronaut sleep intensity [19]

5) Calculate the probability of taking a sleep medication based on the sleep intensity from the SAFTE output, and execute a bootstrap decision for sleep medication utilization.

A Monte Carlo approach was used to integrate these functions over the defined parameter space.

At the core of the ability to assess the need for sleep aids is the ability to generate realistic sleep/work schedules for ISS missions and to use those to calculate the level of sleep intensity, a measure of the need for sleep.

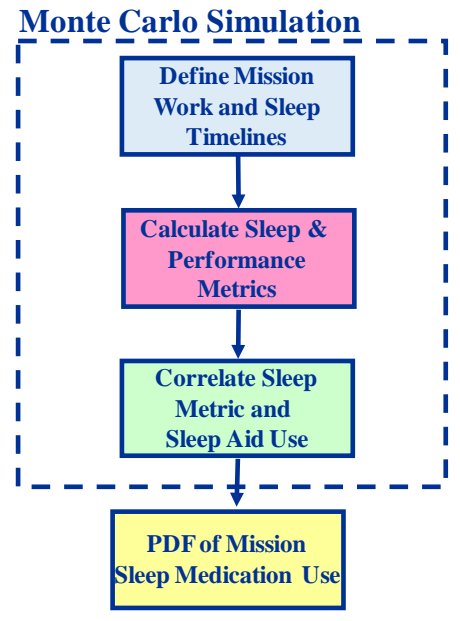

Figure 4. Illustration of the conceptual framework for predicting sleep disruption resulting in the need for a medical intervention.

The mission parameter module generates realistic ISS mission schedules of sleep and wake times. The sleep/wake schedules include events that cause a shift in the sleep schedule (Shuttle, Soyuz and Progress Dockings and Extra Vehicular Activities) and were derived from historical planned and actual ISS mission schedules (Personal Communication with Lauren Leveton, 2008). The best available data on measured sleep patterns from past US space missions was used to model the actual amount of sleep obtained by astronauts in space and the quality of that sleep [20;21]. The quality of sleep depends upon the frequency and quantity of wakefulness, or interruptions, during a sleep period.

The effects of sleep timing and duration and the influence of time of day (circadian effects) on performance are estimated using a deterministic model, modulated by the input parameters of the random schedule generator. The Sleep, Activity, Fatigue, and Task Effectiveness (SAFTE) Model, (IBR, Inc.) [19] was identified as an accurate and wellestablished integration tool. This model was chosen for five reasons:

1) It predicts performance in non-arbitrary units of performance effectiveness that relate directly to performance on a psychomotor vigilance task.

2) It requires a minimum number of input variables.

3) It has a method for reflecting the degrading effects of sleep fragmentation or sleep interruptions.

4) It tracks changes in circadian phase based on changes in the work/rest schedule that commonly occur on ISS.

5) It has been validated as an accurate predictor of risk in the work environment [22].

At the center of the model is a sleep reservoir which, along with the circadian rhythm and sleep inertia, determines performance effectiveness. With some minor modifications SAFTE was easily incorporated into our modeling approach to 
provide estimates of sleep intensity for every sleep opportunity for a given ISS mission schedule.

As defined in our general approach to modeling and in the estimate of wrist fracture probability, a functional transformation relation was needed to assess sleep aid use requirements from the estimate of sleep intensities. In this case, direct evidence of sleep aid use on ISS cannot be determined from observed data, as medication use for this condition is effectively voluntary and not explicitly tracked. In this case, estimates from subject matter experts at the Air Force Research Laboratory and Institutes for Behavior Resources, Inc. were used to estimate terrestrial based threshold levels suitable for defining the translation formulation.

Figure 5 illustrates the predictive results from 5,000 Monte Carlo trials of a simulated ISS increment utilizing the mission parameter program, with its associated distributions, and also distributions for sleep structure and sleep quality., In this case, the mean sleep medication use was predicted to be $51 \pm 39(95 \% \mathrm{CI})$. For a six person crew, this corresponds to approximately 306 sleep aids per ISS increment. Estimates of this type are critical in the design and maintenance of the on orbit medical kit.

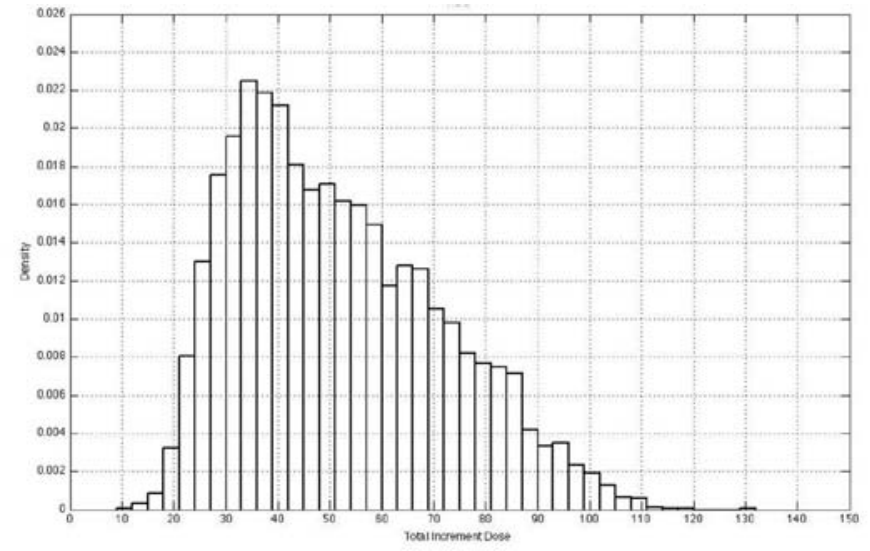

Figure 5. Illustration of predicted sleep aid medication requirements per astronaut per ISS mission, incorporating shifts in the schedule and assuming terrestrial performance measures.

\section{DISCUSSION AND CONCLUSIONSS}

The need to estimate the likelihood of medical risk during space missions is quite evident. However the small number of space faring participants and the limited exposure time severely curtails using estimates of likelihood based solely on the frequency of the observed phenomena. In this effort a process based on well established PRA practices, augmented to address the unique responses of clinical events and their contributors, has been established and shown to produce informative assessments of critical mission scenarios.

The ISS wrist fracture module predicts a relatively low nominal occurrence of traumatic fractures due to translational impacts on ISS, which qualitatively agrees with assessments of medical operations physicians. The model provides a much needed integration of the best available data for estimating the likelihood of a significant risk to crew and mission. It should be noted that the prediction uncertainty, as defined by the standard deviation, is relatively high and directly influences subsequent predictions of crew evacuation within the IMM model.

The application of the modeling process to estimate the quantity of sleep aid medication use with respect to the specific ISS mission parameters represents a unique application of this form of risk modeling. Such efforts can directly benefit mission planners, the assessment of treatment strategies by medical operations personnel and the optimization of the in-flight medical kits. The critical limitation in this case is the lack of data that correlates the predicted sleep intensities with actual sleep aid use. Efforts to utilize research data to supplement in-flight clinical observations are underway to improve the predictive ability of this module.

All modeling efforts require some form of validation and assessment of model credibility, and this is especially true of models that are used for decision making and mission planning. Each of these models is validated on a component basis, i.e. the components are rigorously validated and the integrated module is validated as data becomes available. Both the wrist fracture module and SDMIF have been used to estimate likelihoods from simplified terrestrial scenarios with acceptable success. Details of the validation process for these and other modules produced by our groups will be the subject of future publications.

In conclusion, the use of a probabilistic approach, augmented from well established PRA practices, has been used to estimate the likelihood of fractures and sleep medicine use for astronauts on typical ISS missions. The models use well accepted deterministic modeling approaches to act as integrators of observed physiology, performance level and environment, which can contribute to the clinical risk. Such data fills a critical void in the assessment of risks to astronaut health and mission success.

\section{REFERENCES}

[1] E. Kerstman, Minard C, Saile L, deCarvalho MF, Myers J, Walton M, Butler D, Iyengar S, Johnson-Throop K, and Baumann D, "The Integrated Medical Model: A Risk Assessment and Decision Support Tool for Space Flight Medical Systems," AsMA Annual Meeting, Phoenix, AZ, 2010.

[2] E. S. Nelson, B. Lewandowski, A. Licata, and J. G. Myers, "Development and validation of a predictive bone fracture risk model for astronauts," Ann Biomed Eng, vol. 37, no. 11, pp. 2337-2359, Nov.2009.

[3] J. Cordey, "Introduction: basic concepts and definitions in mechanics," Injury, vol. 31 Suppl 2, p. S-13, May2000.

[4] A. M. Briggs, A. M. Greig, J. D. Wark, N. L. Fazzalari, and K. L. Bennell, "A review of anatomical and mechanical factors affecting vertebral body integrity," International Journal of Medical Sciences, vol. 1, no. 3, pp. 170-180, 2004.

[5] W. C. Hayes and E. R. Myers, "Biomechanical considerations of hip and spine fractures in osteoporotic bone," Instr. Course Lect., vol. 46, pp. 431-438, 1997. 
[6] A. LeBlanc, V. Schneider, L. Shackelford, S. West, V. Oganov, A Bakulin, and L. Voronin, "Bone mineral and lean tissue loss after long duration space flight," J Musculoskelet Neuronal Interact, vol. 1, no. 2, pp. 157-160, Dec.2000.

[7] L. J. Melton, III, S. Khosla, S. J. Achenbach, M. K. O'Connor, W. M. O'fallon, and B. L. Riggs, "Effects of body size and skeletal site on the estimated prevalence of osteoporosis in women and men," Osteoporos. Int., vol. 11, no. 11, pp. 977-983, 2000.

[8] C. Wu, D. Hans, Y. He, B. Fan, C. F. Njeh, P. Augat, J. Richards, and H. K. Genant, "Prediction of bone strength of distal forearm using radius bone mineral density and phalangeal speed of sound," Bone, vol. 26, no. 5, pp. 529-533, May2000.

[9] A. R. Amir and D. J. Newman, "Research into the effects of astronaut motion on the spacecraft: a review," Acta Astronaut., vol. 47, no. 12, pp. 859-869, Dec.2000.

[10] Conway B.A., "Development of Skylab Experiment T-013 Crew/Vehicle Disturbances," National Aeronautics and Space Administration, Washington DC,NASA TN D-6584, 1972.

[11] Klute G.K., "Pilot investigation: nominal crew induced forces in zeroG,"SAE Technical Paper 921155, 1992.

[12] D. J. Newman, A. R. Amir, and S. M. Beck, "Astronaut-induced disturbances to the microgravity environment of the Mir Space Station," J. Spacecr. Rockets., vol. 38, no. 4, pp. 578-583, July2001.

[13] L. Stirling, K. Willcox, P. Ferguson, and D. Newman, "Kinetics and kinematics for translational motions in microgravity during parabolic flight," Aviat. Space Environ. Med., vol. 80, no. 6, pp. 522-531, June2009.

[14] K. M. DeGoede and J. A. shton-Miller, "Fall arrest strategy affects peak hand impact force in a forward fall," J. Biomech., vol. 35, no. 6, pp. 843-848, June2002.

[15] K. M. DeGoede, J. A. shton-Miller, A. B. Schultz, and N. B. Alexander, "Biomechanical factors affecting the peak hand reaction

\section{BIOGRAPHIES}

Dr. Jerry G. Myers, Jr.

NASA Glenn Research Center

21000 Brookpark Road, MS 77-7

Cleveland, OH 44135

Email: Jerry.G.Myers@nasa.gov

Phone: (216) 433-2864

Dr. Myers currently works at the NASA Glenn Research Center, Human Research Program Office in Cleveland Ohio. Dr Myers leads and manages GRC's efforts in computational physiology and probability risk assessment to quantify the risk for evaluating the physiological and medical effects of space travel. Dr. Myers also leads much of the validation and credibility assessment of simulations models used by NASA's Human research program.

Dr. Beth E. Lewandowski

NASA Glenn Research Center

21000 Brookpark Road, MS 110-3

Cleveland, OH 44135

Email: Beth.E.Lewandowski@nasa.gov

Phone: (216) 433-8873

Beth Lewandowski is an employee of the NASA Glenn Research Center and works as a Biomedical Engineer in the Bioscience and Technology Branch. Dr. Lewandowski engages in research of spaceflight induced muscle atrophy and bone loss and the impact of those losses on astronaut health, safety and performance. She utilizes ground based analog data and spaceflight data in the development of computational simulations of various mission scenarios to perform her research.

Mr. John E. Brooker force during the bimanual arrest of a moving mass," J. Biomech. Eng, vol. 124, no. 1, pp. 107-112, Feb.2002.

[16] J. Lo, G. N. McCabe, K. M. DeGoede, H. Okuizumi, and J. A. shtonMiller, "On reducing hand impact force in forward falls: results of a brief intervention in young males," Clin. Biomech. (Bristol. , Avon. ), vol. 18, no. 8, pp. 730-736, Oct.2003.

[17] R. A. Scheuring, C. H. Mathers, J. A. Jones, and M. L. Wear, "Musculoskeletal injuries and minor trauma in space: incidence and injury mechanisms in U.S. astronauts," Aviat. Space Environ. Med., vol. 80, no. 2, pp. 117-124, Feb.2009.

[18] P. L. Davidson, D. J. Chalmers, and S. C. Stephenson, "Prediction of distal radius fracture in children, using a biomechanical impact model and case-control data on playground free falls," J. Biomech., vol. 39, no. 3, pp. 503-509, 2006.

[19] S. R. Hursh, D. P. Redmond, M. L. Johnson, D. R. Thorne, G. Belenky, T. J. Balkin, W. F. Storm, J. C. Miller, and D. R. Eddy, "Fatigue models for applied research in warfighting," Aviat. Space Environ. Med., vol. 75, no. 3 Suppl, p. A44-A53, Mar.2004.

[20] D. J. Dijk, D. F. Neri, J. K. Wyatt, J. M. Ronda, E. Riel, C. A. RitzDe, R. J. Hughes, A. R. Elliott, G. K. Prisk, J. B. West, and C. A. Czeisler, "Sleep, performance, circadian rhythms, and light-dark cycles during two space shuttle flights," Am. J. Physiol Regul. Integr. Comp Physiol, vol. 281, no. 5, p. R1647-R1664, Nov.2001.

[21] Frost JD, Shumate WH, Salamy JG, and Booher CR, "Experiment M133, Sleep Monitoring on Skylab," in Biomedical Results from Skylab. Johnston RS and Dietlein LF, Eds. Washington, DC: NASA, 1997.

[22] Hursh SR, Raslear TG, Kaye AS, and Franzone JF, "Validation and calibration of a fatigue assessment tool for railroad work schedules, summary report," U.S. Department of Transportation, Washington, DC,DOT/FRA/ORD-06/21, 2006.

NASA Glenn Research Center

21000 Brookpark Road, MS 77-5

Cleveland, OH 44135

Email: John.E.Brooker@nasa.gov

Phone: (216) 433-6543

John Brooker is an Aerospace Engineer in the Space Processes and Experiments Division at the NASA Glenn Research Center in Cleveland, $\mathrm{OH}$.

Dr. Aaron S. Weaver

NASA Glenn Research Center

21000 Brookpark Road, MS 110-3

Cleveland, OH 44135

Email: Aaron.S.Weaver@nasa.gov

Phone: (216) 433-3757

Aaron Weaver is a Biomedical Engineer in the Bioscience and Technology Branch at the NASA Glenn Research Center in Cleveland, OH. Dr. Weaver's research interests include the effect microgravity on bone loss and fracture healing, probabilistic modeling of injury for space exploration, and the development of technologies to protect spaceflight crew health and safety. 


\section{Assessing the Likelihood of Rare Medical Events in Astronauts}

Despite over half a century of manned space flight, the space flight community is only now coming to fully assess the short and long term medical dangers of exposure to reduced gravity environments.

Further, as new manned spacecraft are designed and with the advent of commercial flight capabilities to the general public, a full understanding of medical risk becomes even more critical for maintaining and understanding mission safety and crew health. To address these critical issues, the National Aeronautics and Space Administration (NASA) Human Research Program (HRP) has begun to address the medical hazards with a formalized risk management approach by effectively identifying and attempting to mitigate acute and chronic medical risks to manned space flight.

This paper and presentation describes NASA Glenn Research Center's (GRC) efforts to develop a systematic methodology to assess the likelihood of in-flight medical conditions. Using a probabilistic approach, medical risks are assessed using well established and accepted biomedical and human performance models in combination with fundamentally observed data that defines the astronauts' physical conditions, environment and activity levels. Two different examples of space flight risk are used to show the versatility of our approach and how it successfully integrates disparate information to provide HRP decision makers with a valuable source of information which is otherwise lacking. 
ASSESSING THE LIKELIHOOD OF RARE MEDICAL EVENTS IN ASTRONAUTS

Thursday, January 27, 2011, 10:00 AM - 12:15 PM

\author{
Jerry G. Myers, Jr., Ph.D NASA GRC \\ Beth E. Lewandowski, Ph.D NASA GRC \\ John Brooker, NASA GRC \\ Aaron Weaver, Ph.D NASA GRC
}

National Aeronautics and Space Administration (NASA)

John H. Glenn Research Center (GRC) 


\section{Integrated Medical Model (IMM)}

Likelihood of occurrence, probable severity of occurrence, and optimization of treatment and resources.

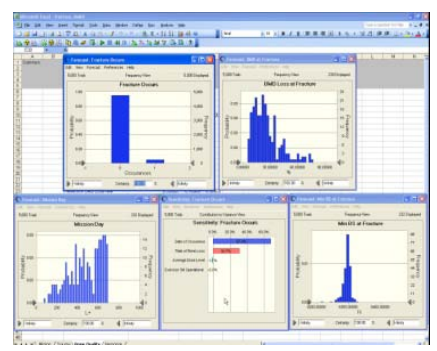

- Probability and consequences of medical risks

- Integrate best evidence in a quantifiable assessment of risk

- Identify medical resources necessary to optimize health and mission success 


\section{IMM and External Modeling}

- Forecasting rare medical concerns confounded by space travel

- Insufficient data (few or no occurrences)

- No clear correlation to terrestrial analog

- Examples

- Traumatic injury

- Skeletal fracture

- Renal stone

- Sleep medication use

- Hypothesis

- Much like an engineering problem, higher fidelity models can provide guidance in assessing medical incidence risk

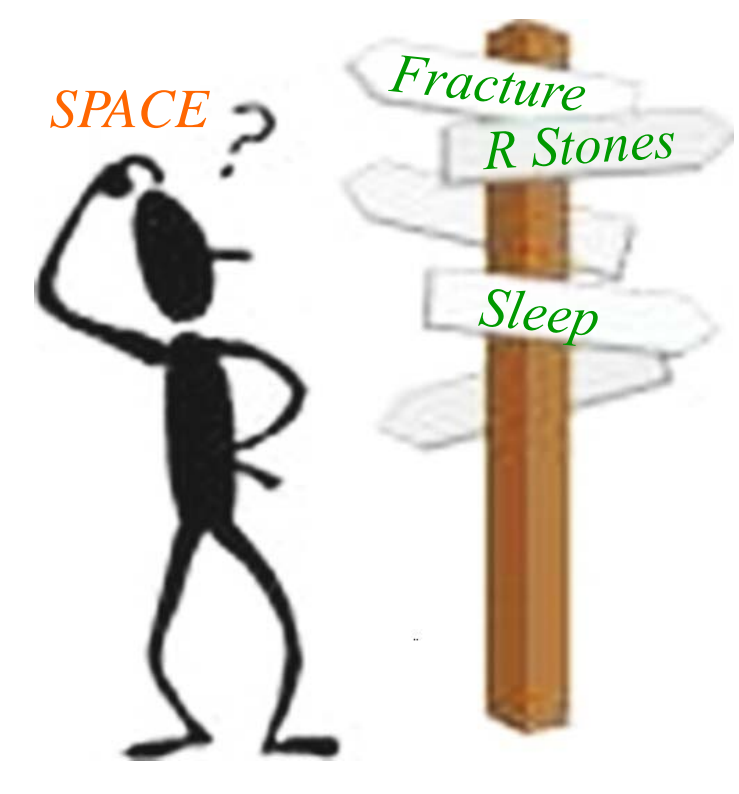

- Requires

- Acceptable level of model maturity

- Proper integration of model and observed data 
"There are no new ideas. There are only new ways of making them felt." Audre Lorde

- Process for Model Development

- Formulate the problem

- Clinical event of concern

- Context of space mission

- Collect information / data

- Primary medical event metrics

- Biomechanics, physiology or performance models

- Parameter distributions

- Construct and validate model

- PRA (Monte Carlo) model to estimate medical event likelihood

- Component and whole model validation
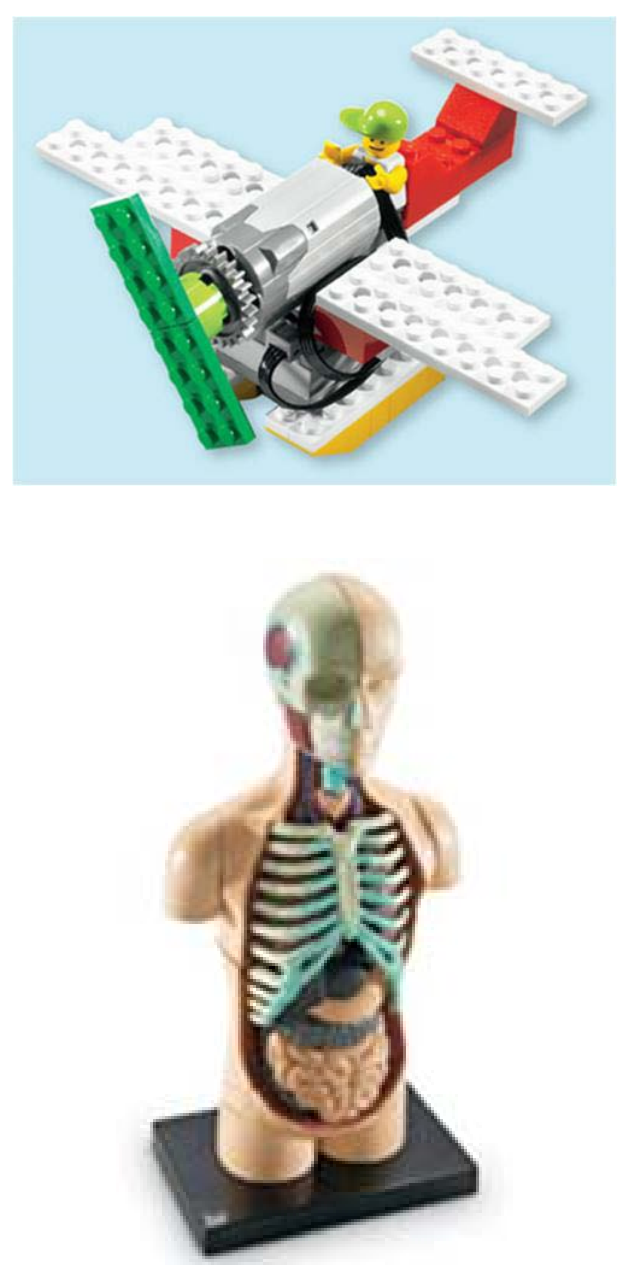

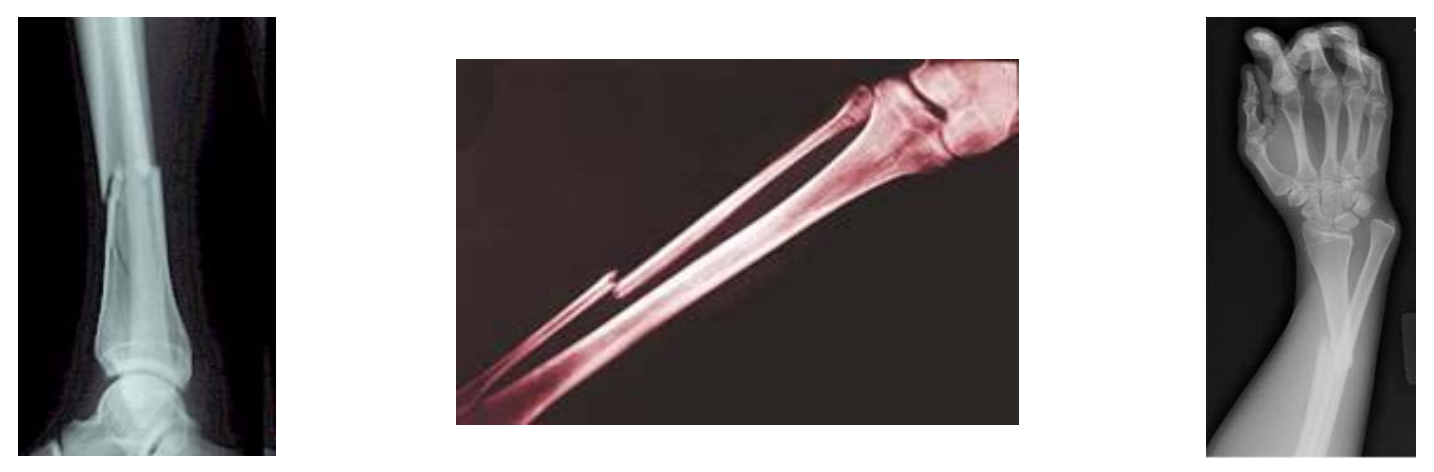

Lead By Example If You Expect Success

\section{EXAMPLE: LIKELIHOOD OF ISS WRIST FRACTURE}




\section{Formulate the Problem: In Flight Fracture Risk}

- Real and Present Concern: Skeletal Fracture

- Weakened Bones

- Off-nominal loading states

- Lack of In Flight Injuries

- Even at areas of high bone loss

- spine and proximal femur

- Fracture risk at wrist

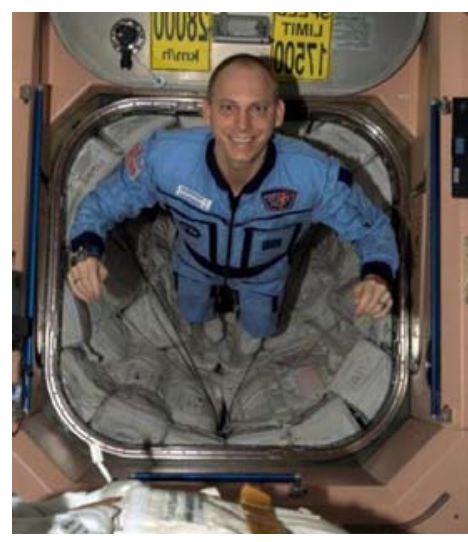

- Some non-fracture in-flight injuries

- Many types suggested translation type injuries

- Load level and rate uncertain

- What is the fracture risk during a push off, impact or other sudden deceleration involving the wrist and shoulder after

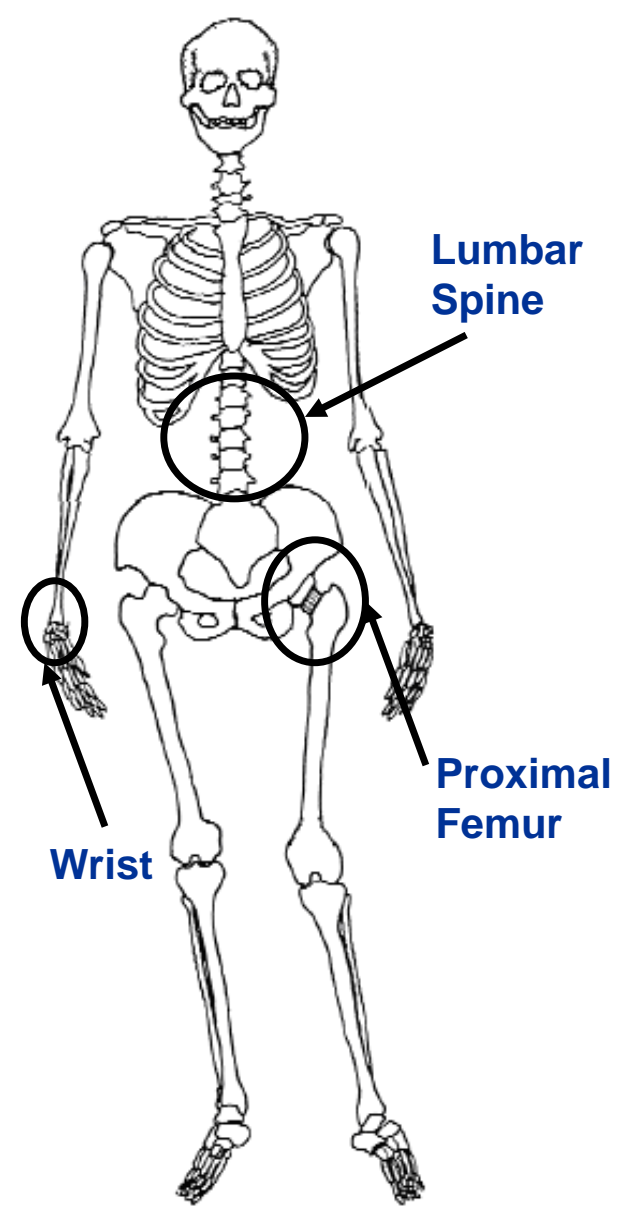
translation? 


\section{Collect Information and Design Model}

- Estimate the probability of loading event occurrence during each mission day

- Estimate the skeletal strength distribution at the wrist

- Skeletal loading

- mass-spring-damper, biomech model of the wrist, arm and shoulder

- Identify distributions of all parameters

- Transfer function that translates FRI to a probability of fracture

- FRI = Loading/ Skeletal strength
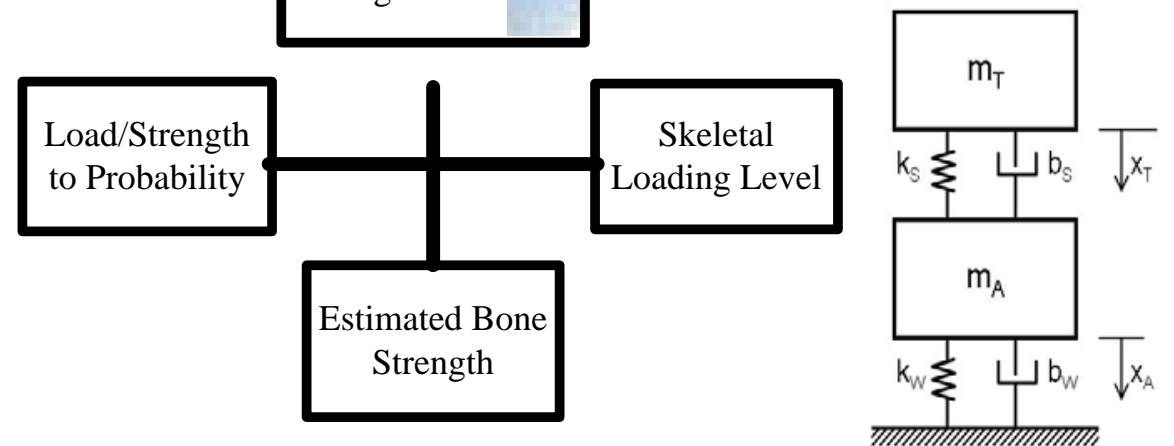

- Monte Carlo simulation to integrate model and data components

- Output is a PDF of the probability of fracture per mission 


\section{Wrist Fracture Probability Estimates}
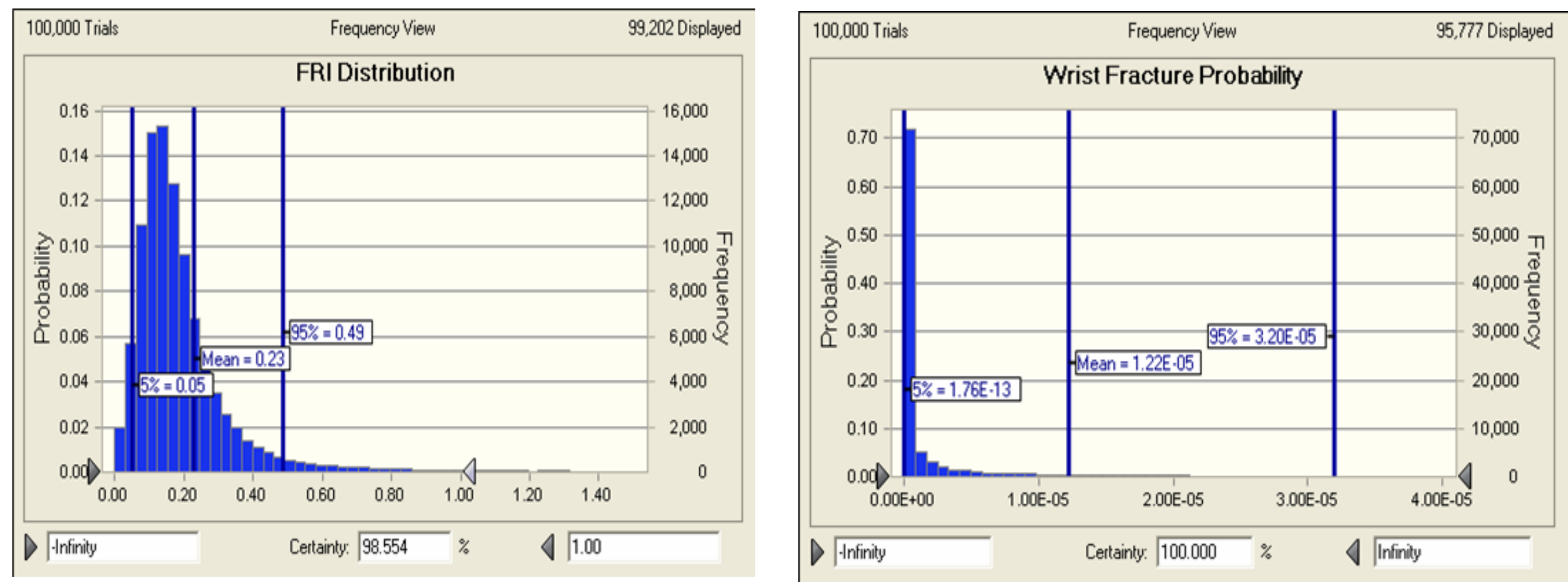

Probability of wrist fracture is relatively low, but not insignificant Qualitatively agrees with the Med-Ops Physician assessments 

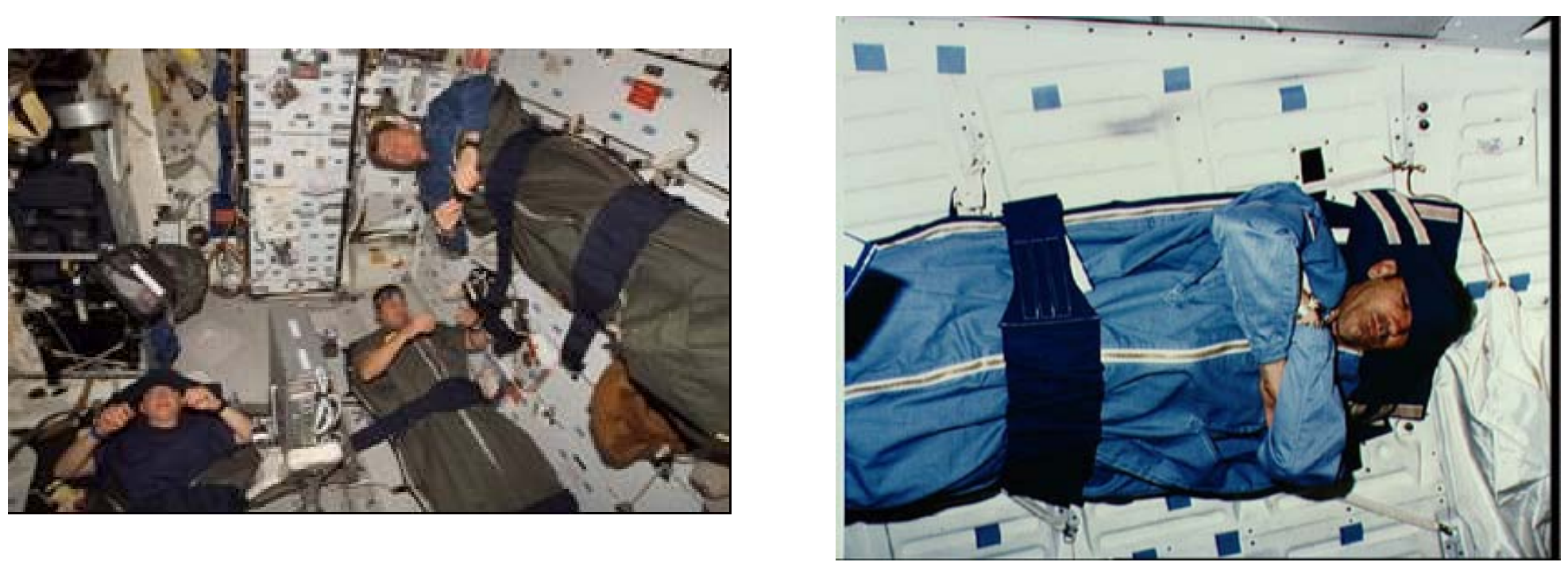

Lead By Example If You Expect Success

\section{EXAMPLE: RATE OF SLEEP AID NEED ON ISS}




\section{Formulate the problem: Space Flight and Sleep}

- Space flight induces reductions in sleep

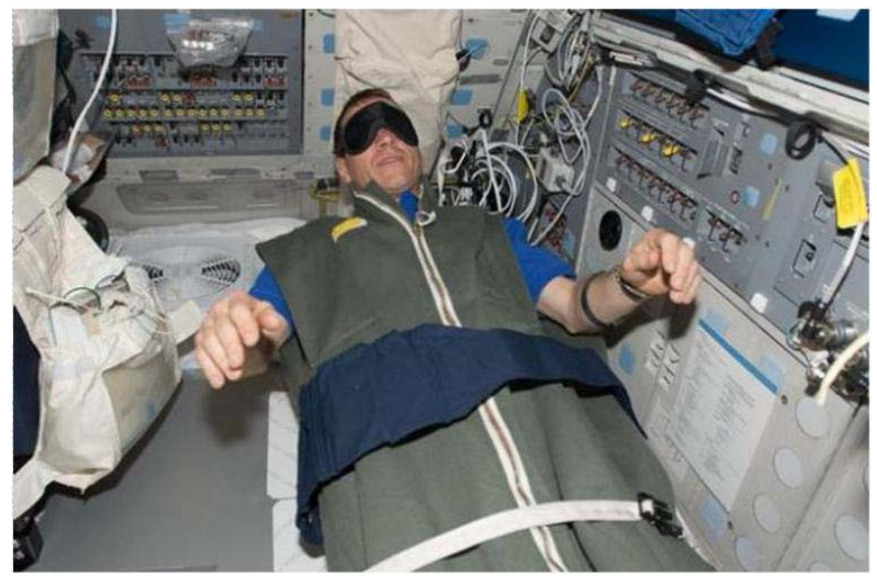
quality and quantity

- Potential performance decrements

- Primary mitigation

- Consolidated and efficient sleep

- Power naps

- Extended sleep opportunities

- Impacted by scheduling and environmental factors

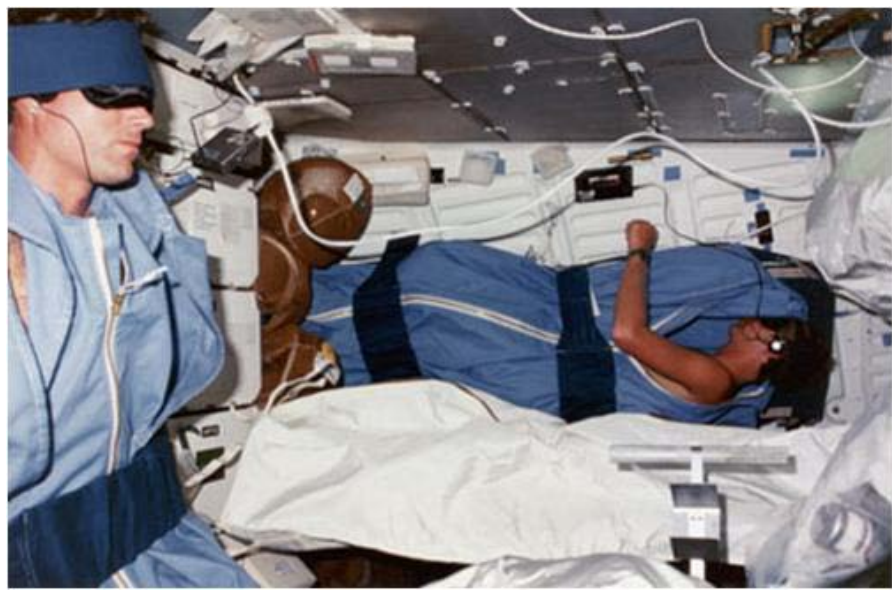

- Medications or sleep aids

- Medication usage rates not explicitly known

- Worse Case ISS Mission

- $6 \mathrm{crew}, 2$ pills a night, 0.5 years $=2 \mathrm{~K}$ doses

- What are the rates of medical intervention resulting from low sleep quality and duration? 


\section{Sleep Disruption- Medical Intervention Forecasting (SDMIF) Tool}

- Derive realistic daily schedule

- Crew sleep opportunities

- Crew sleep time

- Sleep quality

- Utilize validated modeling of sleep and performance

- SAFTE proprietary process

- Accurately reflects the degrading effects of changes in circadian phase, sleep fragmentation or sleep interruptions

- Calculate sleep intensity from the SAFTE output

- Execute a bootstrap decision for sleep medication utilization

- Translation function derived from Air Force Research Laboratory and

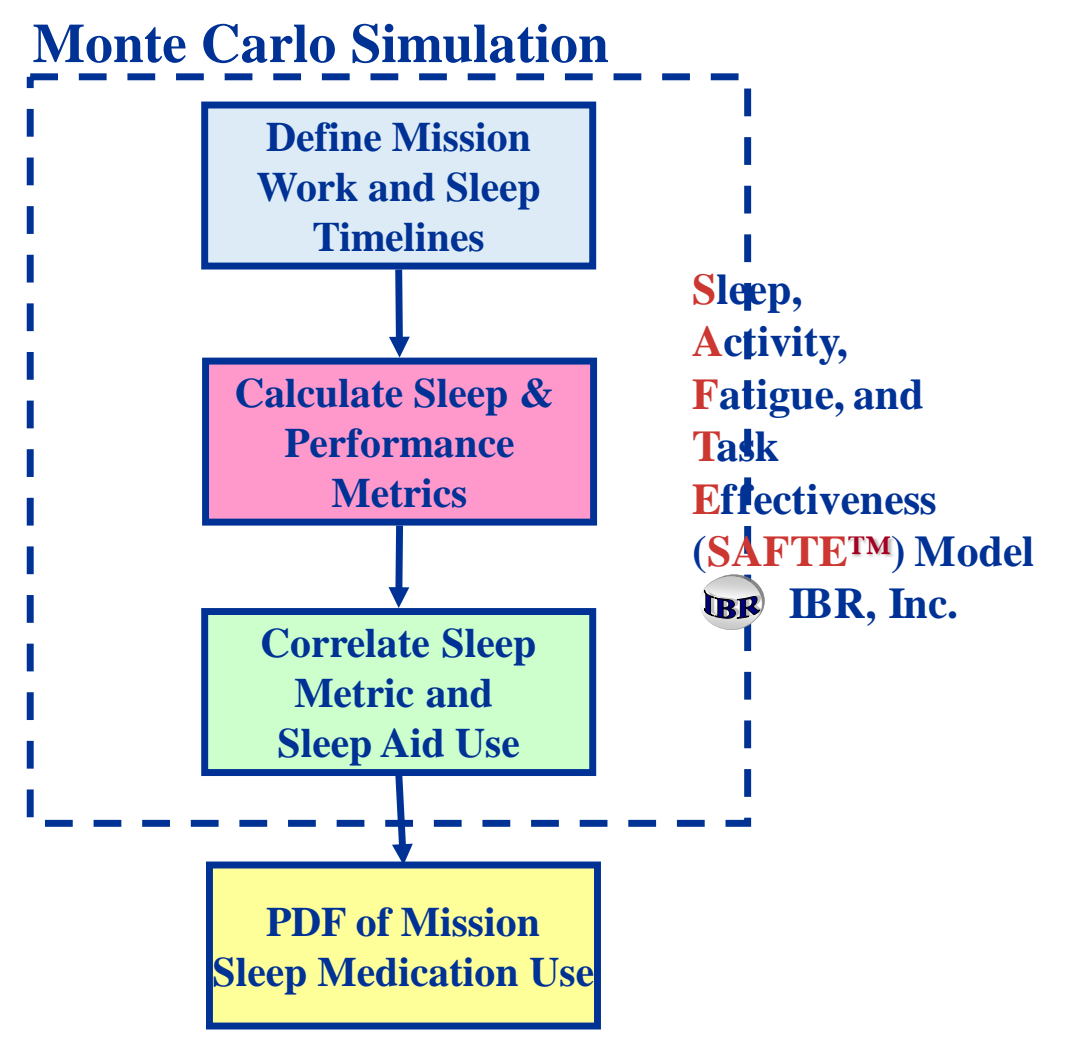
Institutes for Behavior Resources, Inc. subject matter experts 


\section{SDMIF Example Output for an ISS Mission}

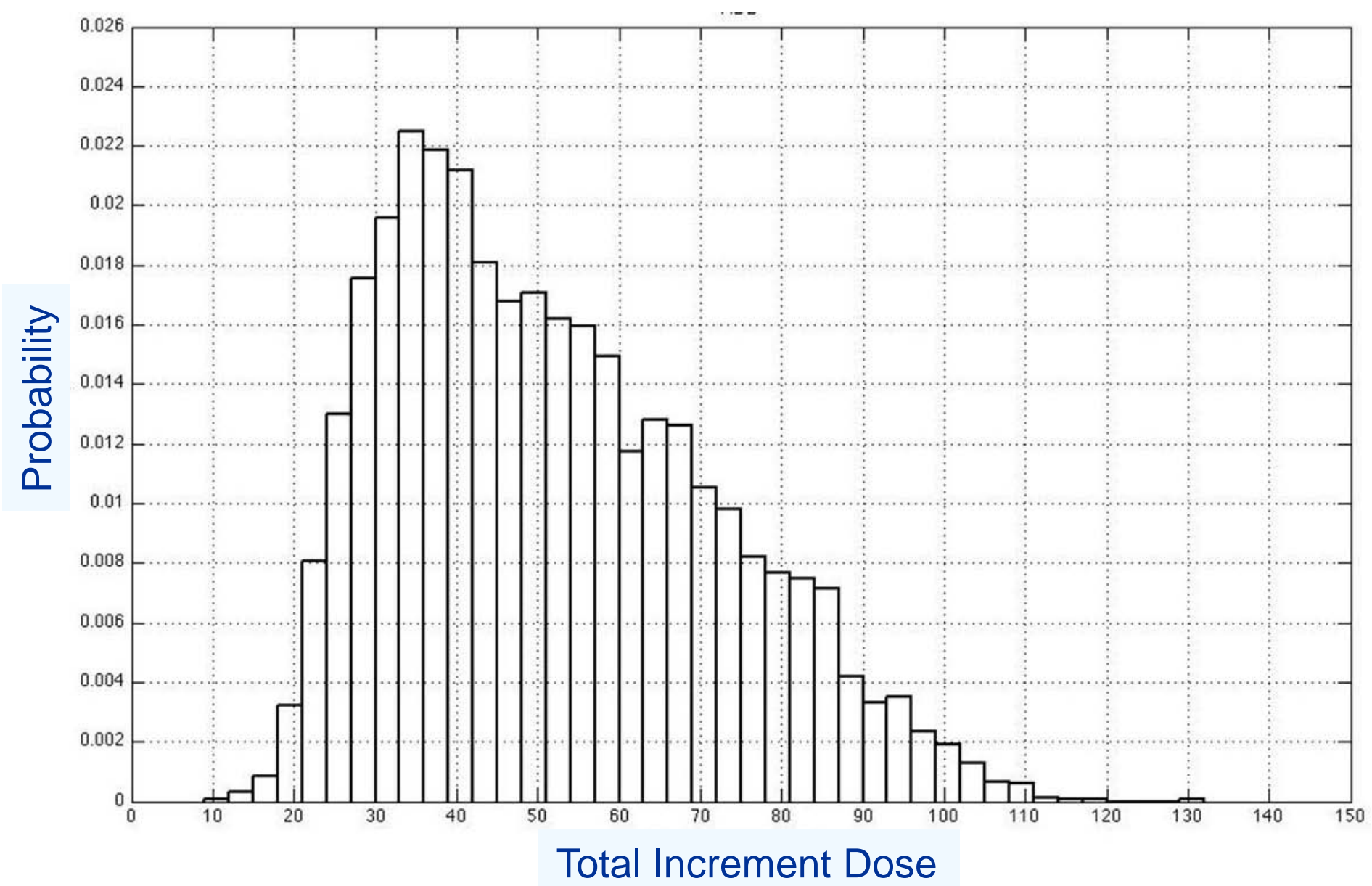

Estimated mean rate of sleep aid use is $1 / 3^{\text {rd }}$ that of the WCS estimate 


\section{Assessing Acceptability of the Models}
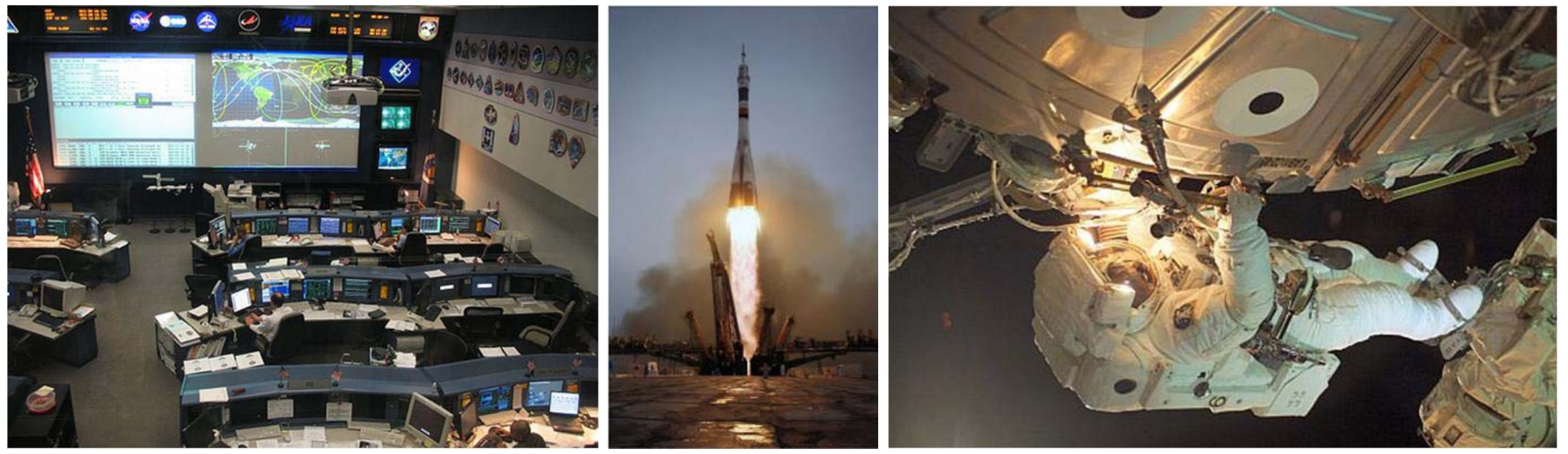

- Chief factor is an acceptable V\&V process

- Complicated by limited in-flight data

- Relies on systematic use of SME's and multiple validation approaches to balance limits in direct comparative evidence

- Models related to medical events used to inform Space Flight Operations and Planning

- Must exhibit a high level of Credibility for the intended use

- Must have their Credibility assessed over multiple factors 


\section{V\&V? Its Really About Model Credibility!}

Achieving a high level of belief or trust in the model

- NASA-STD-7009

- Standard for Models and Simulations (M\&S)

- M\&S Development

- Verification

- Validation

- M\&S Operations

- Input Pedigree

- Results Uncertainty

- Results Robustness

- Supporting Evidence

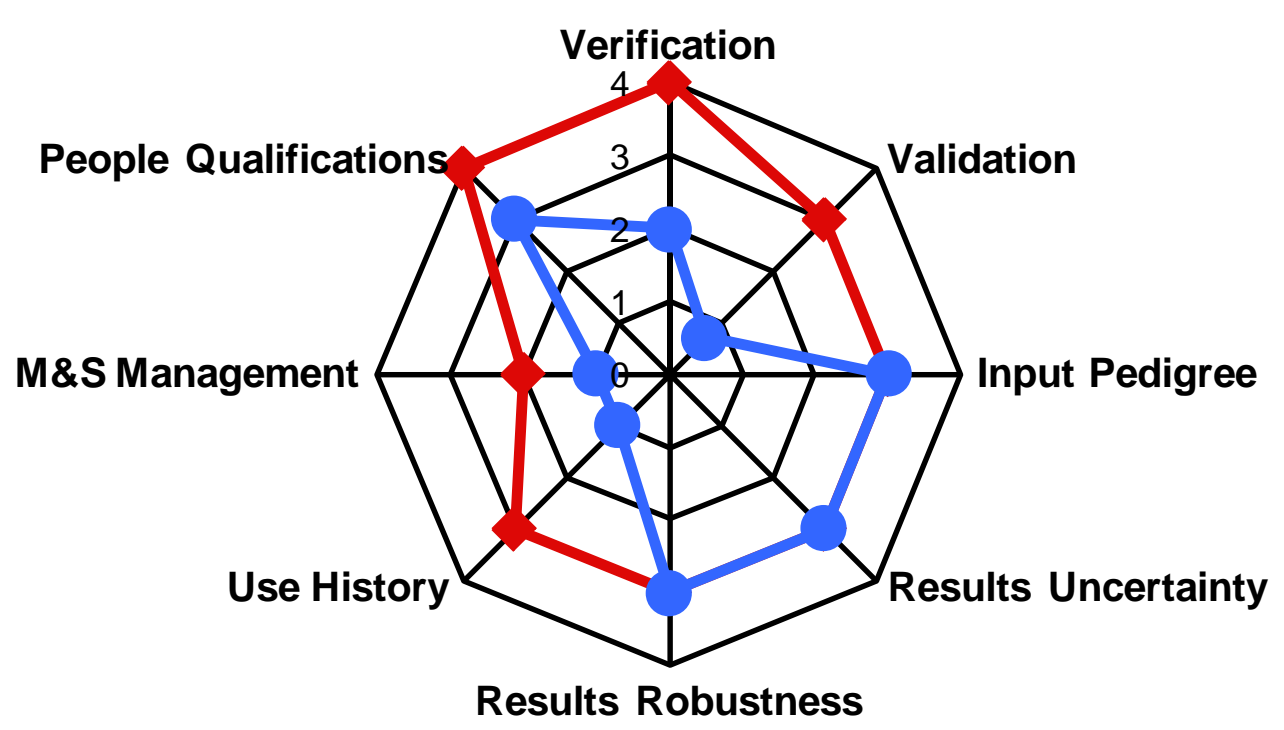

- Use History

- M\&S Management

- People Qualifications

Sufficiency Thresholds

Factor Score

Both the Wrist Fracture Model and SDMIF are undergoing component wise and full function credibility assessment 


\section{Let's Review}

- IMM utilizes external modules to supplement observed data

- Two examples: Bone Fracture and Sleep Disruption

- Others

- Renal stones, head and neck injuries

- External modules

- Utilize PRA concepts and tools to develop higher fidelity probabilistic models

- Development invokes

- Flexible modeling practices

- Well vetted existing models

- Concepts to address confounding contributions to the risk

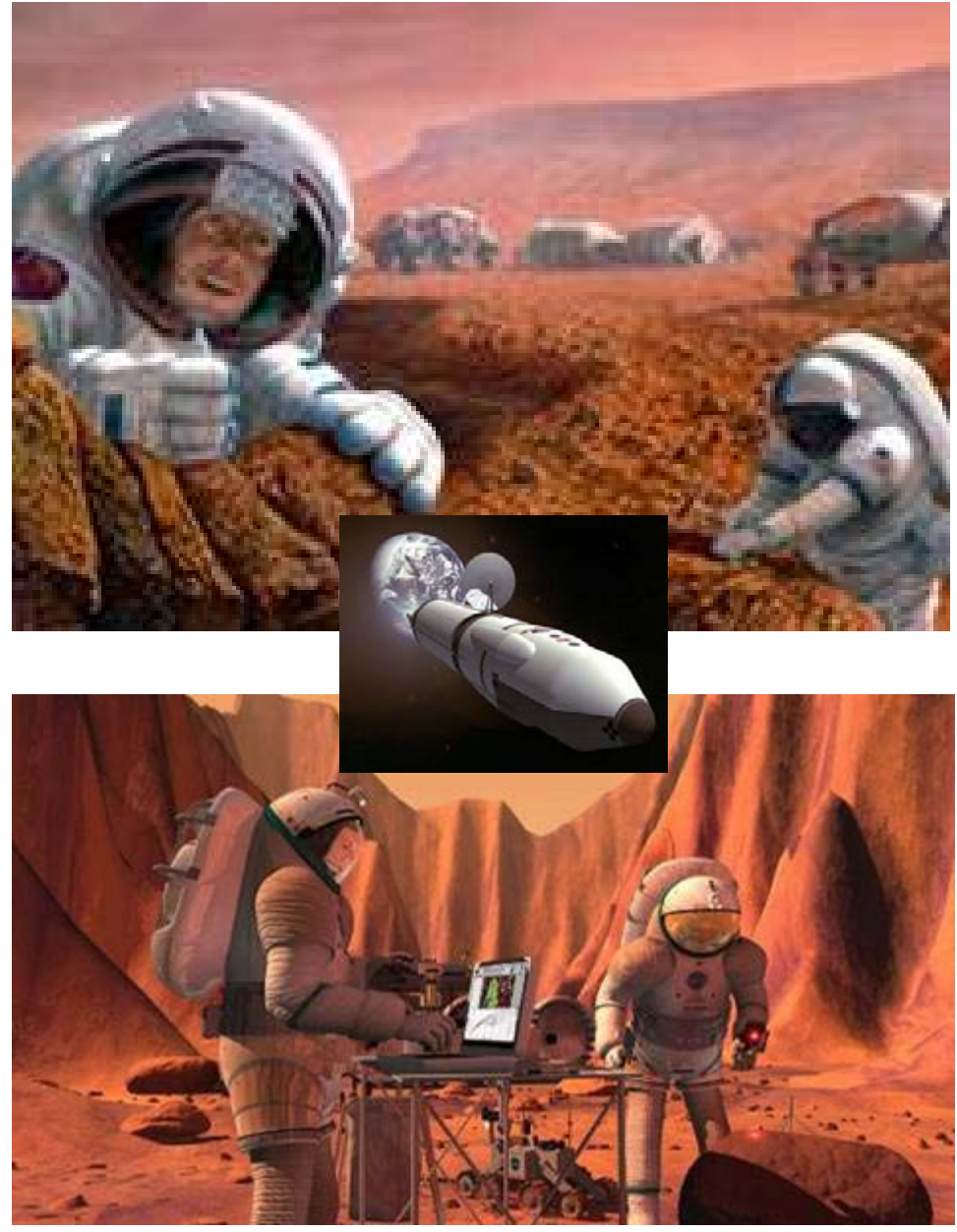


"The most important questions of life are indeed, for the most part, really only problems of probability." - Pierre Simon Laplace

\section{QUESTIONS}

